\title{
Improve Static Performance Identification Of Inductive Proximity Sensor For a Mobile Robot
}

\author{
Nguyen Hoai and Nguyen Le Chau Thanh \\ Department of Mechanical Engineering, College of Engneering, College of Technology. The University of Danang, Da Nang, Viet Nam
}

\begin{abstract}
Nowadays, inductive proximity sensor and mobile robot are one of the most interesting topics attracting scientists. With enhancement techniques from using sensor for controlling mobile robots which can be developed more in the future. Additionally, measuring of static performance of inductive proximity sensor is also essential because the testing and navigation systems perform more exactly by result information from the experiment by assaying the output signal. In this paper, the hand-made measurement system is used to verify again the static performance of proximity sensor with ferrous, non-ferrous and alloy metal tape. Simultaneously, improve static properties of typical tape metal through the relationship between the output signal and the sensing distance of sensor. According to theoretical basis, the relationship of the static parameters can be determined such as: thickness of metal, sensing distance, dimension of metal, and so on. The testing results have shown the output signal of proximity sensor in the model and theory is not similar. The final data will accommodate some information about the relationship of the thickness of metal tape and the sensing distance of proximity sensor, the different affects of position sensor and optimum operation range of mobile robot.
\end{abstract}

\section{Introduction}

The inductive proximity sensor is a common sensor to search a metal guideline for a mobile robot [1]. It uses to detect metallic objects without touching them. The fundamentals of the non-contacting inductive proximity sensor (IPS) and achieved status in year 2002 of the miniaturization of IPS resulting from the high integration level and utilization of modern assembly technologies were presented in [2].

IPS operates on the principle of the inductance change due to change in magnetic field. It detects the presence and absence of the ferrous and nonferrous metal objects without physically touching the object itself. If a metallic object moves into the field, the resulting induced currents add load to the oscillator, stopping oscillation [3]. In fact, when inductive proximity sensor analyzes the position of metal objects needed to be detected by a decrease in the quality of the oscillator circuit's electromagnetic [4], some unknown objects arise under the main tape which is put above the obstruction sheet. This is one of many reasons making noise in the sensor signal [5]. The relationship.

between the sensing distance of proximity sensor and the thickness of the metal is shown in Figure 1.

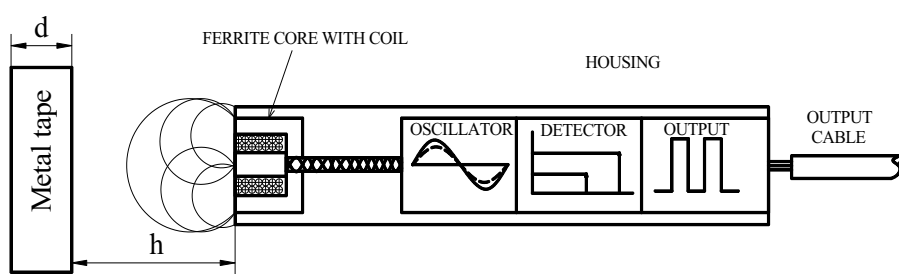

Figure 1. The inductive proximity sensor configuration

Although many kinds of different proximity sensor being developed but its specifications have yet to meet the capabilities of mobile robot control. Therefore, to measure the static performance of an inductive proximity sensor is the key technology to improve the performance of a mobile robot [6]. In this study, static performance testing is the determination of the sensing distance (h) and object thickness (d), continuously by moving the sensor from the edge of beginning to end of metal and go out of it. Finally, the results are analyzed and collated to enhance the continued trend for the control of a mobile robot by using the inductive proximity sensor and also using demands of the industrial process.

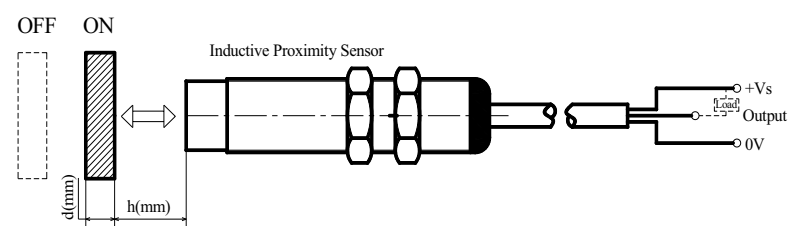


Figure 2. Measurement of sensing distance (h) and thickness of the metal tape (d)

\section{Testing set-up of measurement system}

A testing set-up measurement system is shown in Figure 3. Inductive proximity sensor is held again rotation, but allowed to move up and down. To measure sensing distance, using height gauge is fixed to the sensor. Each sensor has three wire, in which blue wire is connected to the negative power supply, brown wire is connected to the positive power supply through resistor $(\mathrm{R}=10 \Omega)$, and the black wire is the signal wire. The power supply is DC power. To observe and compare the output signal of the sensor, connect the negative wire (blue wire) with the oscilloscope. When an inductive proximity sensor moves into the sensing area and sensor activities, the output sign turns ON. Otherwise, an inductive proximity sensor moves out of the sensor sensing area, the output sign turns OFF. To determine static performance of sensor, many kind of metallic objects, such as: steel, aluminum, copper are chosen.

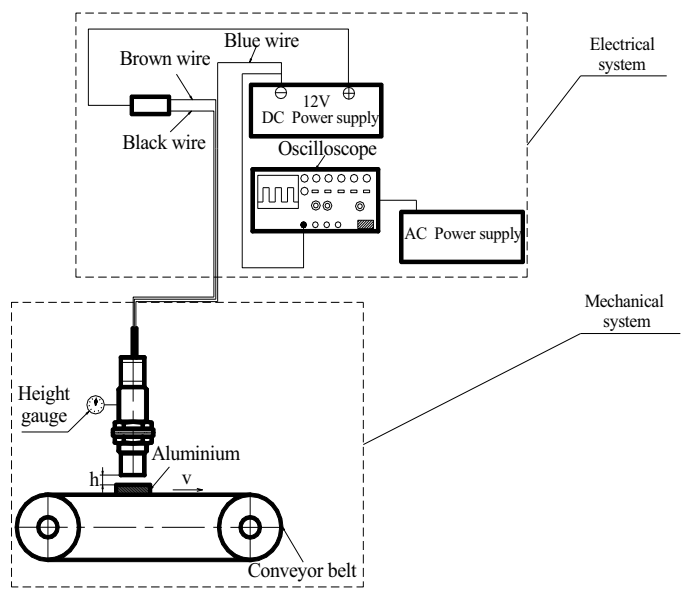

Figure 3. Diagram of measurement system with digital proximity sensor

From result determining the sensing distance of aluminum, keeping the constant sensing distance and move the sensor along the length of the aluminum tape, using measurement equipment (as a Figure 4). Corresponding to the each a value, the shape of the signal (Vavg) will change.

Here: $\mathrm{s} 1$ is the smallest distance, in which, the signal shape began to change

$\mathrm{s} 2$ is the largest distance, in which, the signal shape changes more than any initial position and the amplitude virtually unchanged over most of the length of the aluminum tape.
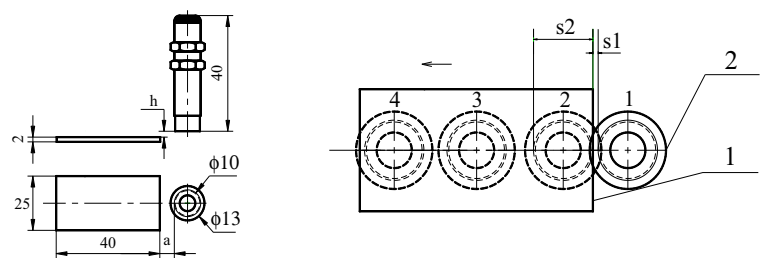

Figure 4. A testing set-up of measurement system for improvement static performance

\section{Result and discussion}

The curves of the sensing distance (h) depend on materials of ferrous, non-ferrous and alloy. 301 stainless steel is ferrous with excellent corrosion resistance, so the shape of the graph is different to copper and aluminum. With this result, the identification of sensor in the range between $0.06 \mathrm{~mm}$ to $0.18 \mathrm{~mm}$ thickness is easier than in the range between $0.24 \mathrm{~mm}$ to $0.5 \mathrm{~mm}$ thickness. When using sensor to measure detecting distance of aluminum and copper, the shape of curves is similar.

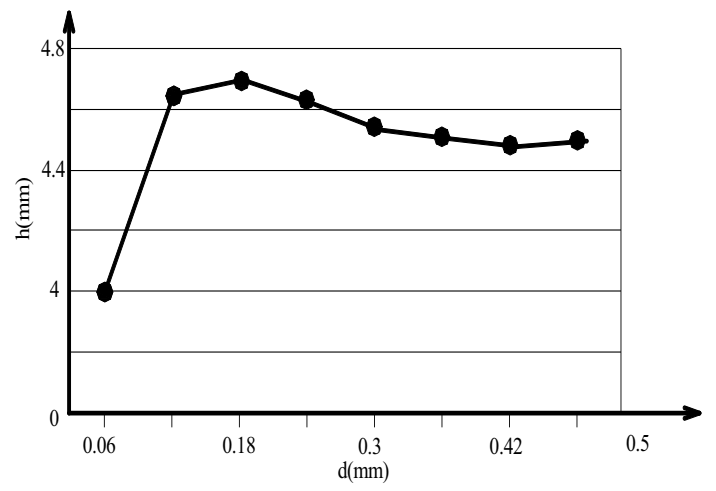

(a)301 Stainless steel

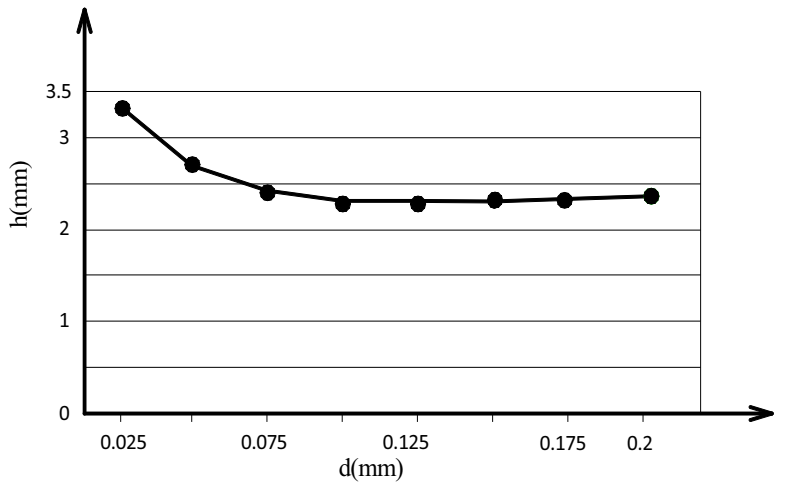

(b)Copper

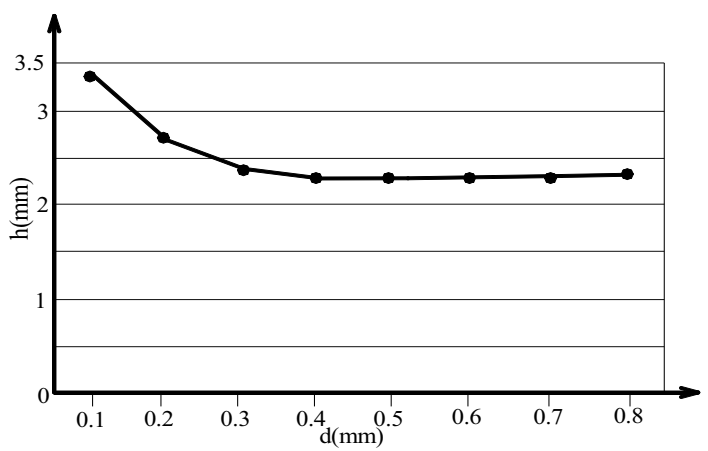

(c)Aluminium 


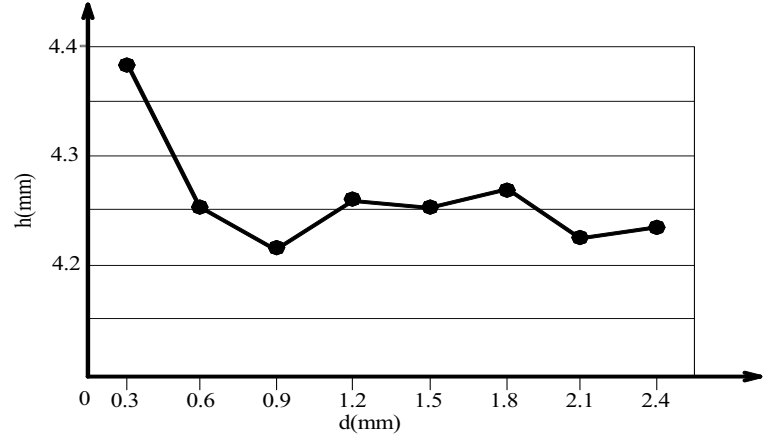

(d) Alloy

Figure 5. Relationship of sensing distance and thickness of objects

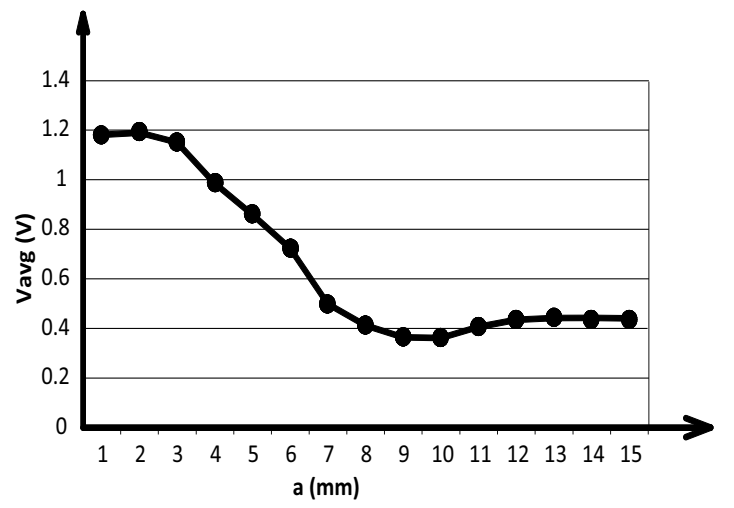

(a) $\mathrm{h}=0.5 \mathrm{~mm}$

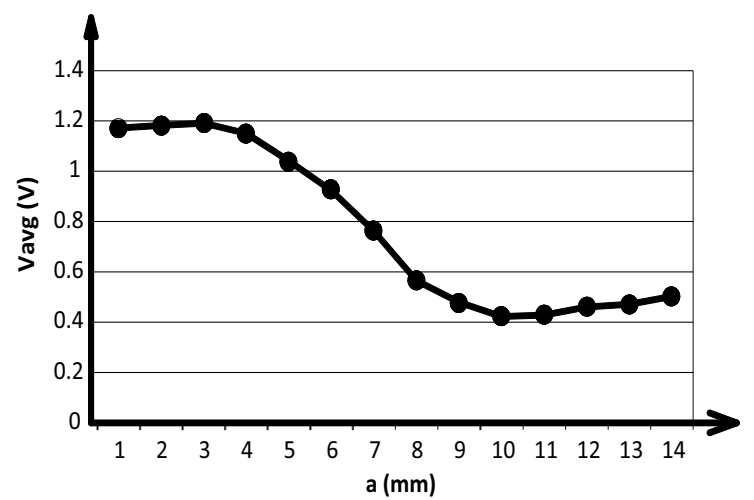

(b) $\mathrm{h}=0.7 \mathrm{~mm}$

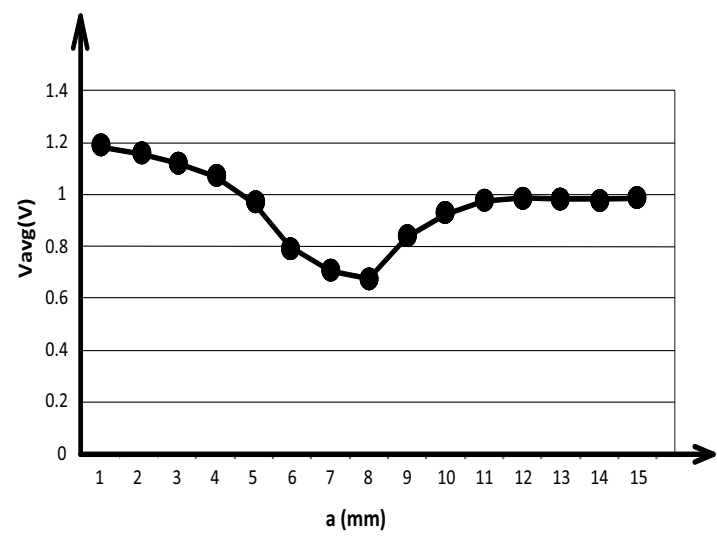

(c) $\mathrm{h}=1.5 \mathrm{~mm}$

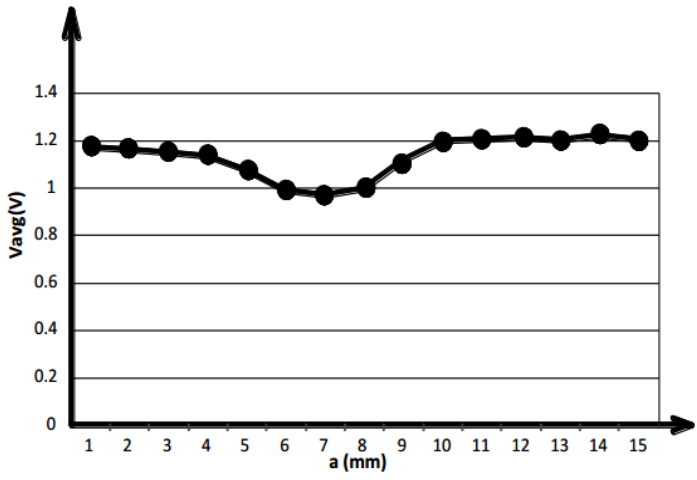

(d) $\mathrm{h}=2.5 \mathrm{~mm}$

Figure 6. Relationship of parameters: h,s, $V_{\text {avg }}$

This can be explained by the same attributes (nonferrous). In these cases, the identification of sensors is easy and quickly from $0.025 \mathrm{~mm}$ to $0.125 \mathrm{~mm}$ thickness copper and from $0.1 \mathrm{~mm}$ to $0.4 \mathrm{~mm}$ thickness aluminum. Continuously, with alloy tape, the shape of graph is similar with copper and aluminum's measurement. This is explained by the alloy component contains non-ferrous metals (Cu-Al alloy). So, it is difficult to determine the optimal identification of the proximity sensor for alloy.

In the Figure 6.a-d, with $\mathrm{h}=2.5 \mathrm{~mm}$ (as shown in Fig.6d), the value of the constant average voltage can't be seen, even when moving the sensor into the aluminum tape. Specifically, $V_{\text {avg }}$ will increase and exceed the initial value at $\mathrm{a}=10 \mathrm{~mm}(1.2 \mathrm{~V})$.

The sensing distance with range from $0.5 \mathrm{~mm}$ to 0.7 $\mathrm{mm}$, the received signal is consistent with theoretical knowledge (as shown in Fig.6a, b). When changing a distance from $9 \mathrm{~mm}$ to $15 \mathrm{~mm}, \mathrm{~V}_{\text {avg }}$ hardly changed. This is the basis for a control mobile robot. In addition, with the smaller sensing distance, the faster initial signal change. With $\mathrm{h}=1.5 \mathrm{~mm}, \mathrm{~V}_{\text {avg }}$ changes from $1.17 \mathrm{~V}$ to $1 \mathrm{~V}$ (a $=1-4 \mathrm{~mm}$ ), while $\mathrm{h}=0.5 \mathrm{~mm}$, Vavg change from 1.17 $\mathrm{V}$ to $0.39 \mathrm{~V}(\mathrm{a}=1-4 \mathrm{~mm})$.

\section{Conclusion}

In summary, for the static performance of proximity sensor, the signals of metal tape that the signals of ferrous, non-ferrous and alloy metal tape have giant differences, as indicated from the experimental results. when the material is ferrous metal, there exists a period of initial thickness of the material, in which the sensing distance is proportional to it. Otherwise, when the material is nonferrous metal, there exists a period of initial thickness of the material, in which the sensing distance is inversely proportional to it. Additionally, when measuring in the same materials, sensing distance doesn't depend on the initial thickness of that material. This is the key to improve the performance of a mobile robot.

Besides, distance of above $0.7 \mathrm{~mm}$ is not only the biggest distance for mobile robot to control more accurate in the process but only more sensitive than other distance.

\section{Reference}


1. T.T.Tsung and H.Nguyen, "Measurement of static performance of inductive proximity switch for a mobile robot", Applied Mechanics and Materials, vol.404, pp.502-507, 2013.

2. M.Jagiella and S.Fericeam, "Miniaturized inductive sensors for industrial applications", in Sensors, Proceedings of IEEE, pp.771-778, 2002.

3. R.C. Luo, "Sensor technologies and micro-sensor issues for mechatronics systems",

4. Mechatronics, IEEE/ASME Transactions on, vol.1, pp.39-49, 1996.

5. J.Fraden, Handbook of modern sensors: physics, designs, and applications: Springer, 2004.

6. T.T.Tsung, T.T.K.Vy, and N.Hoai, "The Disturbance of Inductive Proximity Sensor for Mobile Robot", Advanced Materials Research, pp.909-912, 2015.

7. T.T.Sung, T.T.K.Vy, and N.Hoai, "Testing and Improvement of Static Performance of Proximity Sensor for Mobile Robot", Key Engineering Materials, vol.656-657, pp. 719-724,2014. 